\title{
\begin{tabular}{l|l} 
Mibraries & DSpace@MIT
\end{tabular}
}

\author{
MIT Open Access Articles
}

\section{High Temporal and Spectral Resolution Interferometric Observations of Unusual Solar Radio Bursts}

The MIT Faculty has made this article openly available. Please share how this access benefits you. Your story matters.

Citation: Oberoi, D., E. R. Evarts, and A. E. E. Rogers. “High Temporal and Spectral Resolution Interferometric Observations of Unusual Solar Radio Bursts." Solar Physics 260.2 (2009) : 389-400. Copyright (C) 2009, Springer Science+Business Media

As Published: http://dx.doi.org/10.1007/s11207-009-9459-x

Publisher: Springer

Persistent URL: http://hdl.handle.net/1721.1/65384

Version: Author's final manuscript: final author's manuscript post peer review, without publisher's formatting or copy editing

Terms of Use: Article is made available in accordance with the publisher's policy and may be subject to US copyright law. Please refer to the publisher's site for terms of use. 


\title{
High Temporal and Spectral Resolution Interferometric Observations of Unusual Solar Radio Bursts
}

\author{
D. Oberoi ${ }^{1}$, E.R. Evarts ${ }^{1,2,3}$, A.E.E. Rogers ${ }^{1}$, \\ ${ }^{1}$ Massachusetts Institute of Technology, Haystack Observatory, Westford, MA 01886, \\ U.S.A. (doberoi@haystack.mit.edu) \\ 2 Dept. of Physics, Brandeis University, 415 South Street, Waltham, MA 02454, U.S.A. \\ 3 Dept. of Physics, Carnegie Mellon University, 5000 Forbes Avenue, Pittsburgh, PA \\ 15213, U.S.A.
}

Received ; accepted

\begin{abstract}
.
We report very high temporal and spectral resolution interferometric observations of some unusual solar radio bursts near $1420 \mathrm{MHz}$. These bursts were observed on 13 September 2005, 22 minutes after the peak of a GOES class X flare from the NOAA region 10808. Our observations show 11 episodes of narrow-band intermittent emission within a span of $\approx 8 \mathrm{~s}$. Each episode shows a heavily frequency-modulated band of emission with a spectral slope of about $-245.5 \mathrm{MHz} \mathrm{s}^{-1}$, comprising up to 8 individual blobs of emission and lasts for $10-15 \mathrm{~ms}$. The blobs themselves have a spectral slope of $\approx 0 \mathrm{MHz}$ $\mathrm{s}^{-1}$, are $\approx 200-250 \mathrm{kHz}$ wide, appear every $\approx 400 \mathrm{kHz}$ and last for $\approx 4-5 \mathrm{~ms}$. These bursts show a brightness temperatures in the range $10^{12} \mathrm{~K}$, which suggests a coherent emission mechanism. We believe these are the first high temporal and spectral resolution interferometric observations of such rapid and narrow bandwidth solar bursts close to 1420 $\mathrm{MHz}$ and present an analysis of their temporal and spectral characteristics.
\end{abstract}

Keywords: Sun: radio bursts, Sun: flares, Sun

\section{Introduction}

The radio emission from the Sun can be rather complex. It shows variations over a range of temporal scales ranging from sub ms to solar cycles and a wide variety of spectral scales. In addition, its large angular size and complicated emission morphology conspire to make radio imaging of the Sun difficult. Consequently most radio observations of the Sun come from single dish instruments. These observations can provide exquisitely detailed frequencytime evolution of the solar emission, but cannot provide any information of the morphology or location of emission within the beam of the telescope. There are only a few radio interferometers in operation (Gauribidanur Radioheliograph (40-150 MHz), Nançay Radioheliograph (150-450 MHz), Owens Valley Solar Array (1-18 GHz), Siberian Solar Radio Telescope (5.7 $\mathrm{GHz})$ and Nobeyama Radioheliograph $(17,34 \mathrm{GHz})$ ) which can routinely provide images of solar emission with resolutions ranging from a few to a tenth of an arcmin. These images enable the study of location, morphology and evolution of radio features on solar surface and their correlations with

(C) 2009 Springer Science + Business Media. Printed in the USA. 
a host of images obtained across the electromagnetic spectrum from other instruments.

Interferometric observations offer the additional advantage that they are not sensitive to emission at angular scales larger than the resolution of the baselines involved. Hence the use of sufficiently long baselines resolves out the strong, large angular-scale, and featureless emission of the solar disk and can preferentially pick up weaker compact emission. Pushing this idea to its logical extreme, there have been some attempts to look for extremely compact solar source of emission $(<100 \mathrm{~km})$ by conducting Very Long Baseline Interferometry (VLBI) observations of the Sun (Tapping, 1986; Benz et al., 1996). These observations, conducted at a few $\mathrm{GHz}$ using baselines ranging between 360 and $3800 \mathrm{~km}$ produced mixed results. Using $1.7 \mathrm{GHz}$ observations with 70 mas (milli arcsec) resolution, Tapping (1983) presented evidence for multiple compact sources in an impulsive burst event, while at $2.3 \mathrm{GHz}$ with resolutions ranging between 8 and 90 mas, Benz et al. (1996) reported that none of the 26 radio burst events analyzed had any detectable fringes, consistent with the expectations for scattering of radio waves in the solar corona.

We present very high time and frequency resolution interferometric solar observations of an unusual radio burst at $1.4 \mathrm{GHz}$ with a $\mathrm{km}$ scale baseline. High time and spectral resolution interferometric observations of the Sun are rather rare and the serendipitous discovery of such emission reinforces the notion that this is a relatively poorly explored part of the phase space, a point also made by Gary (2003).

\section{Observational Setup}

A two-element interferometer using $2.3 \mathrm{~m}$ parabolic dishes, operating at a central frequency of $1417.6 \mathrm{MHz}$ was set up at the Haystack Observatory $\left(42.5^{\circ} \mathrm{N}, 71.5^{\circ} \mathrm{W}\right)$. Each dish and its associated electronics were independent Small Radio Telescopes (SRTs) ${ }^{1}$ (Salah et al., 2003). The local oscillators and sampling of the SRTs were controlled by independent GPS receivers at each antenna in the $V L B I$ mode, thereby allowing interferometry. Typical stability performance of the GPS (Trimble 'Thunderbolt') is about $300 \mathrm{ps}$ on a scale of about 30s, which corresponds to $150^{\circ}$ of phase at 1420 $\mathrm{MHz}$. The SRT feeds are sensitive to right circular polarization. Though SRT receiver used an 8-bit ADC to sample the voltage data, in order to keep the data volumes low, only one bit (the sign bit) per sample was captured over a bandwidth of $4 \mathrm{MHz}$ using a sampling rate of 8 Msamples $\mathrm{s}^{-1}$. This data cannot be used to determine total power in the signal, but is quite adequate

\footnotetext{
${ }^{1}$ More information about the Small Radio Telescope can be found at http://www.haystack.mit.edu/edu/undergrad/srt/index.html
} 
for inteferometry and leads to a loss of sensitivity by a factor of $2 / \pi$ (Thompson et al., 1986). A bandwidth-limiting filter restricted the observed radio frequency bandwidth to $\approx 3 \mathrm{MHz}$. The cold sky system temperature, $T_{\text {sys }}$, of the SRT antennas has been measured to be $\approx 100 \mathrm{~K}$ and their aperture efficiency, $\eta$, to be $\approx 50 \%$. This leads to a cold sky inteferometric sensitivity $(1 \sigma)$ of $\approx 80 \mathrm{Jy}$ or $0.008 \mathrm{SFU}\left(1 \mathrm{SFU}=10^{4} \mathrm{Jy}=10^{-22} \mathrm{~W} \mathrm{~m}^{-2} \mathrm{~Hz}^{-1}\right)$ in one second of integration over the $3 \mathrm{MHz}$ observing band. As even the quiet sun is a very intense source and the emission can become even stronger during solar flares, $T_{\text {sys }}$ is much larger when observing the Sun, and the sensitivity proportionally poorer. In the observations presented here, the quiet sun flux at our observing frequency was about $70 \mathrm{SFU}$ (flaring sun flux was around $130 \mathrm{SFU}$ ) (Figure 2), which leads to an antenna temperature of about 540 $\mathrm{K}(1000 \mathrm{~K})$, a system temperature of about $640 \mathrm{~K}(1100 \mathrm{~K})$ and degrades the sensitivity of the interferometer to $0.05 \mathrm{SFU}$ (0.09 SFU) for the same 1 s time integration and $3 \mathrm{MHz}$ bandwidth.

For monitoring purposes, one second averaged total power measured was also recorded at each of the antennas. Due to the limited resources available on the DSP card, only 2 out of every 4096 samples could be used (averaged) to determine the total power. The sensitivity of the total power monitoring system is therefore worse by a factor of $\sqrt{2048}=45.26$, as compared to that for the single dish, and is equal to 2.22 and $3.82 \mathrm{SFU}$, respectively for the case of quiet and bursting sun. The data from the total power monitoring system are succeptible to variations due to a variety of reasons including receiver stability and temperature variations.

The data files from the two SRTs were retrieved and correlated offline using custom software on another PC. Figure 1 shows one of the SRT antennas. The two antennas were separated by $1.4 \mathrm{~km}$ along an azimuth of $20^{\circ}$.

\section{Observations}

The interferometer was operated intermittently from July through September 2005. In the period from 12 to 22 September the SRT interferometer recorded data during every even minute for 50 seconds. During this period the Sun was quite active, the GOES satellite observed several X-ray bursts and several strong radio events were reported by NOAA. In this paper we concentrate on the very unusual event observed on 13 September 2005 at 19:44:10 UT. We note that a very complex sunspot group NOAA region 10808 was active on the Sun during these observations and was close to the center of the solar disc (S09, E10) at the time of this event. According to the event lists provided by Space Environment Center, NOAA, this region was responsible for $47 \mathrm{C}$-class, $20 \mathrm{M}$-class and $10 \mathrm{X}$-class $\mathrm{X}$-ray flares, more 


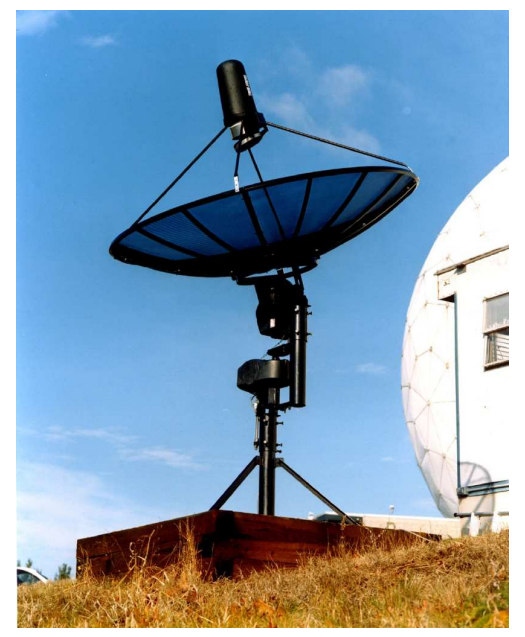

Figure 1. A view of the Small Radio Telescope, an inexpensive educational aid developed at Haystack Observatory to introduce students and amateurs to radio astronomy.

major flares than any other region in the entire 23rd solar cycle. A summary of solar activity recorded around the time of this event is presented in Table I. Our data shows 11 episodes of intermittent narrow-band emission with a large negative spectral slope in a span of $8 \mathrm{~s}$, which are listed in Table II.

The time series of solar flux observed by the total power monitoring system on the two SRT elements is shown in Figure 2. The figure also shows the time series of the solar flux, for the same polarization, central frequency and bandwidth as the SRT observing setup, as observed by the Owens Valley Solar Array (OVSA) $\left(37.23^{\circ} \mathrm{N}, 118.28^{\circ} \mathrm{W}\right)$. There is good agreement, except during the first peak, between the time series of solar flux observed by these instruments. The noise level of the SRT data is consistent with the sensitivity of the total power monitoring system (3.82 SFU). A difference of about 10 SFU is seen in the measurements of the two SRTs in the period 19:44:00 to 19:44:20. Its short lived nature suggests that it was probably due to a small momentary pointing error in one of the SRTs. Given the low sensitivity of the total power monitoring system, this is not particularly signficant.

Though the OVSA and SRT were observing the Sun simultaneously during this event, the OVSA data, unfortunately, does not include observations in the SRT observing band during any of the episodes of emission tabulated in Table II. The OVSA has a rather complicated observing setup and cycles 
Table I. Summary of activity observed on the Sun on 13 September 2005, close to the event presented here. Sagamore Hill (Massachusetts, USA), Palehua (Hawaii, USA) and Holloman (New Mexico, USA) are a part of the Radio Solar Telescope Network operated by United States Air Force $\left(1 \mathrm{SFU}=10^{-22} \mathrm{~W} \mathrm{~m}^{-2} \mathrm{~Hz}^{-1}\right)$

\begin{tabular}{ccccccc}
\hline Instrument & $\begin{array}{c}\text { Start } \\
(\mathrm{UT})\end{array}$ & $\begin{array}{c}\text { Max } \\
(\mathrm{UT})\end{array}$ & $\begin{array}{c}\text { End } \\
(\mathrm{UT})\end{array}$ & Flux & Band & Comment \\
\hline GOES & 1919 & 1927 & 2057 & X 1.5 & X-ray & Region 10808 \\
Sagamore Hill & 1921 & 1923 & 2129 & $24 \mathrm{KSFU}$ & $8.8 \mathrm{GHz}$ & \\
Palehua & 1922 & 1923 & 2130 & $23 \mathrm{KSFU}$ & $8.8 \mathrm{GHz}$ & \\
Palehua & 1922 & 1923 & 2137 & $6.0 \mathrm{KSFU}$ & $2.695 \mathrm{GHz}$ & \\
Sagamore Hill & 1922 & 1923 & 2135 & $4.9 \mathrm{KSFU}$ & $2.695 \mathrm{GHz}$ & \\
Holloman & 1922 & 1923 & 2313 & & H $\alpha$ & Region 10808 \\
SRT & 1944 & & 1944 & & $1.42 \mathrm{GHz}$ & Our observations \\
Palehua & 1950 & & 1951 & & $25-180 \mathrm{MHz}$ & Type III burst \\
Sagamore Hill & 1950 & & 1951 & & $30-180 \mathrm{MHz}$ & Type V burst \\
SOHO/LASCO & 2000 & & & & White light & Halo CME \\
& & & & & & (vel 1866 km s $^{-1}$ ) \\
\hline
\end{tabular}

Table II. Summary of observed parameters of the 11 episodes of emission observed on 13 September 2005.

\begin{tabular}{ccccc}
\hline Episode No. & $\begin{array}{c}\text { Start time } \\
(\mathrm{UT})\end{array}$ & $\begin{array}{c}\text { Spectral } \\
\text { slope } \\
\left(\mathrm{MHz} \mathrm{s}^{-1}\right)\end{array}$ & $\begin{array}{c}\text { Average separation } \\
\text { between peaks } \\
(\mathrm{kHz})\end{array}$ & $\begin{array}{c}\text { No. of emission } \\
\text { blobs in the } \\
\text { episode }\end{array}$ \\
\hline 1 & $19: 44: 10.57$ & -281.4 & 399.2 & 5 \\
2 & $19: 44: 13.13$ & -270.9 & 389.4 & 8 \\
3 & $19: 44: 14.00$ & -245.7 & 400.9 & 7 \\
4 & $19: 44: 14.13$ & -298.2 & 396.8 & 5 \\
5 & $19: 44: 15.03$ & -223.7 & 400.0 & 6 \\
6 & $19: 44: 15.29$ & -219.2 & 400.9 & 7 \\
7 & $19: 44: 16.11$ & -248.8 & 400.9 & 3 \\
8 & $19: 44: 16.17$ & -172.2 & 427.4 & 7 \\
9 & $19: 44: 18.22$ & -255.2 & 393.5 & 8 \\
10 & $19: 44: 18.59$ & -264.6 & 387.1 & 7 \\
11 & $19: 44: 18.64$ & -198.5 & 403.2 & 3 \\
\hline
\end{tabular}




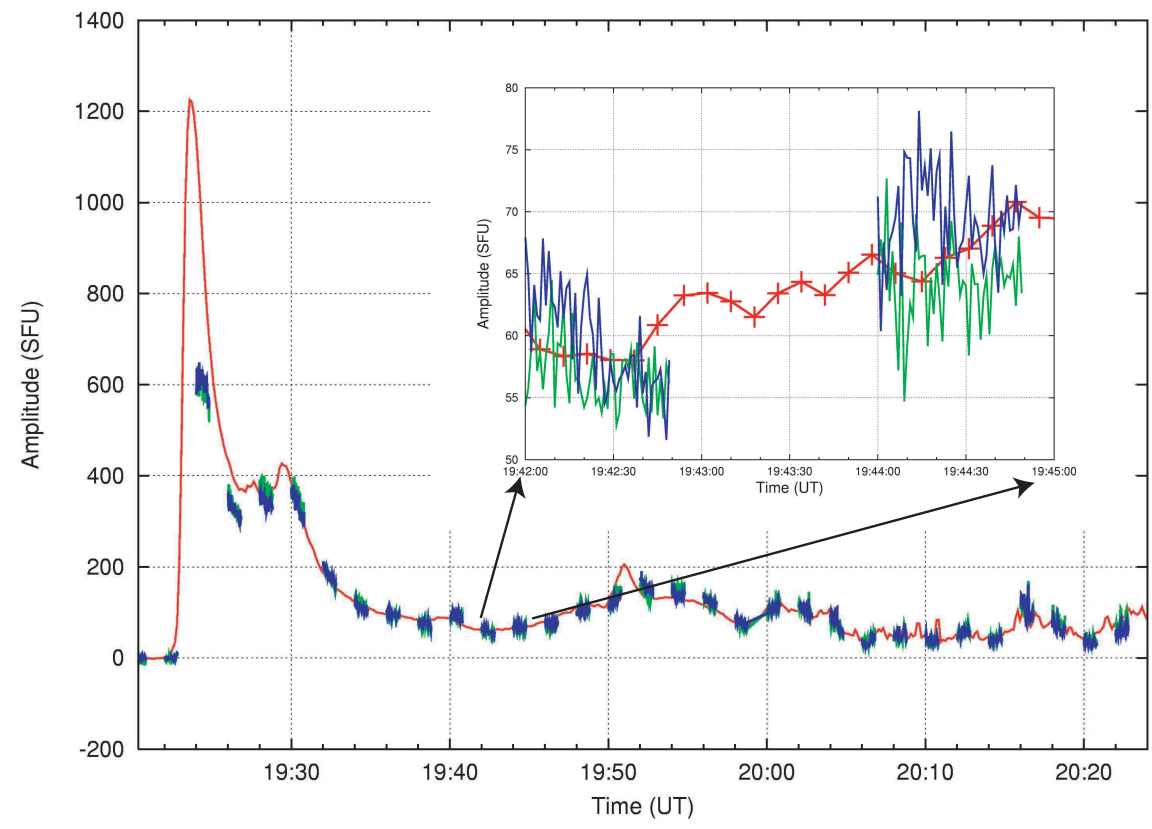

Figure 2. The solar flux light curve provided by the Owens Valley Solar Array (OVSA) for the duration of about an hour in the vicinity of event of interest is shown in red. OVSA routinely subtracts the quiescent pre-flare solar flux level, which in this instance was about 70 SFU. The total power light curve obtained by the two SRTs, scaled to match the flux measured by OVSA, is plotted in green and blue. The inset shows a zoomed-in version of the plot, highlighting the differences between the sampling of the SRT and OVSA data. The $x$-axis shows universal time in hh:mm format for the main figure and in hh:mm:ss format for the inset. The SRT flux is averaged over data spanning one second and the OVSA flux is averaged over $30 \mathrm{~ms}$ with a sampling interval of $8.1 \mathrm{~s}$. The time range of the inset includes the times for the episodes of emission listed in Table II.

through 39 observing frequencies, observing for a duration $70 \mathrm{~ms}$ each at each frequency and polarization. It takes OVSA 8.1s to complete one observing cycle, successive measurements at the same frequency and polarization are hence spaced 8.1s apart. The $1.4 \mathrm{GHz}$ band in the right circular polarization was sampled at OVSA during the $70 \mathrm{~ms}$ period from 19:44:15.200 to 19:44:15.270 which does not overlap with any of the episodes of emission observed with the SRT interferometer.

The episodes of emission observed at 19:44:13.13 UT and 19:44:18.59 UT are shown in Figure 3. The time resolution of the data is $512 \mu$ s and the spec- 

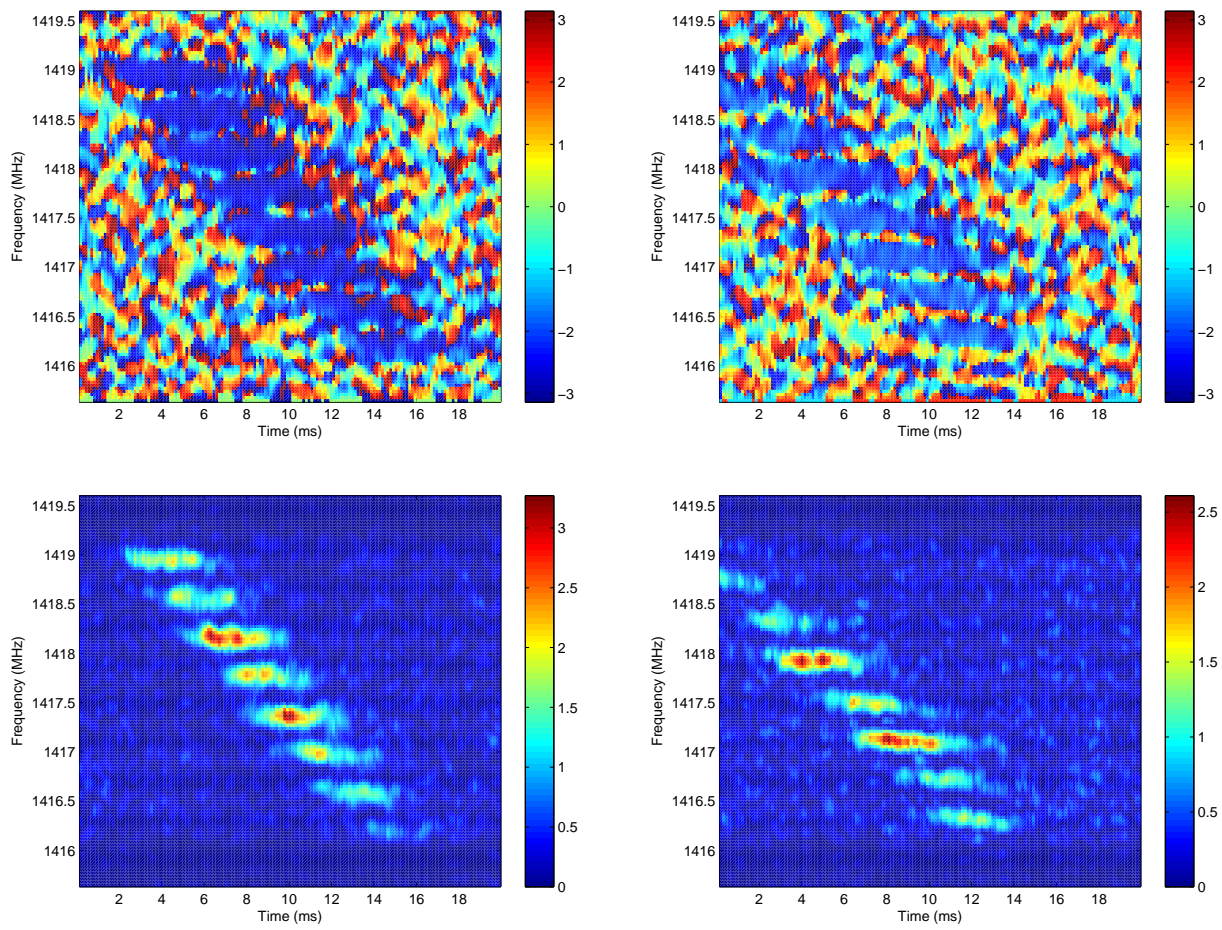

Figure 3. Dynamic spectra for two of the episodes of emission seen at 19:44:13.13 (left panels) and 19:44:18.59 (right panels). Top panels show the phase and the bottom ones the amplitude of the correlations for these episodes of emission. The $x$-axis spans $20 \mathrm{~ms}$ in time. Frequency increases along the $y$-axis and the plot spans $4 \mathrm{MHz}$. Correlation amplitudes are plotted in arbitrary units and the data have not been normalized by the band shape, which is seen as the dark band at the top and bottom of the amplitude panel. The peaks of emission blobs in amplitude plots are $\approx 20 \sigma$. The color scale on the phase panels spans $-\pi$ to $\pi$ radians.

tral resolution is $31.25 \mathrm{kHz}$. One of them shows very similar emission blobs across the entire episode and the other shows a mix of smaller and larger emission blobs. The emission is so heavily modulated in frequency that it has a banded appearance. The blobs do not seem to drift in frequency and are remarkably equally spaced in frequency for a given episode of emission, with the spectral separation varying between $387.1 \mathrm{kHz}$ and $427.4 \mathrm{kHz}$ between different episodes (Table II).

Individual episodes of emission last for 10 to $15 \mathrm{~ms}$ and span $\approx 0.5 \mathrm{MHz}$ to $3 \mathrm{MHz}$, the entire RF span of the system. As the spectral spans of some of the episodes overlap only partially with the observing band, the number of well defined emission blobs in an individual episode range between 3 and 
8. Though it is not as well defined as seen in Figure 3 in a few cases, we can identify the presence of burst emission all through the observing band for 8 of the 11 episodes. This suggests that the intrinsic bandwidth span of these episodes is probably a little larger than the observing bandwidth. A spectral slope was computed for each of these episodes, except one, by fitting a straight line to the leading or the trailing edge of the band. The average spectral slope was $-245.5 \mathrm{MHz} \mathrm{s}^{-1}$ and the distribution of spectral slopes had an $\mathrm{rms}$ of $38.7 \mathrm{MHz} \mathrm{s}^{-1}$. The duration of the individual emissions blobs varied between $\approx 1 \mathrm{~ms}$ and $6 \mathrm{~ms}$ and their spectral width was in the range 125 $\mathrm{kHz}$ to $250 \mathrm{kHz}$ corresponding to a fractional bandwidth of $\approx 10^{-4}$. An upper limit on the instantaneous bandwidth of emission in an episode is $\approx 1.5 \mathrm{MHz}$ (fractional bandwidth $\approx 10^{-3}$ ), so this emission is highly monochromatic.

The projected baseline length at the time of observation was $819.3 \mathrm{~m}$, corresponding to an angular resolution of 53 arcsec or about $3 \%$ of the solar diameter. The fact that the observed phase shows a random distribution except in the regions of emission blobs (Figure 3) implies that the observing baseline has resolved out the emission from the solar disk and the flaring active region.

\section{Analysis}

The total power measurements at each of the SRT antenna-elements are averages over $1 \mathrm{~s}$, and hence are not useful for measuring the flux of individual episodes of emission which last for much shorter durations. Using the light travel time argument to determine the size of the emitting region from

its few ms duration, leads to an upper limit on the source size of order of 1 arcsec, much smaller than the resolution of the SRT interferometer. The OVSA measurements (Figure 2) indicate that the solar flux in the immediate vicinity of these events is about 130 SFU. Using the measured aperture efficiency values of $\approx 50 \%$ for the SRT leads to an antenna temperature, $T_{\text {ant }}$, of $\approx 980 \mathrm{~K}$. Hence, the assumptions of the emission episodes being unresolved by the SRT interferometer and the $T_{\text {sys }}$ being dominated by the solar emission are both well justified. Under these assumptions, the flux of emission episodes is approximately given by

$$
S_{\text {episode }}=\frac{\rho}{1-\rho} S_{\odot}
$$

where $S_{\odot}$ is the solar flux in $\mathrm{W} \mathrm{m}{ }^{-2} \mathrm{~Hz}^{-1}$ before or after the emission episode and $\rho$ is the magnitude of the cross-correlation coefficient observed on the interferometer baseline during the emission episode. Using the 130 SFU value for approximate solar flux and the typical peak $\rho$ of $\approx 0.25$ for the episodes of emission, leads to a typical peak episode flux of $\approx 40 \mathrm{SFU}$, 


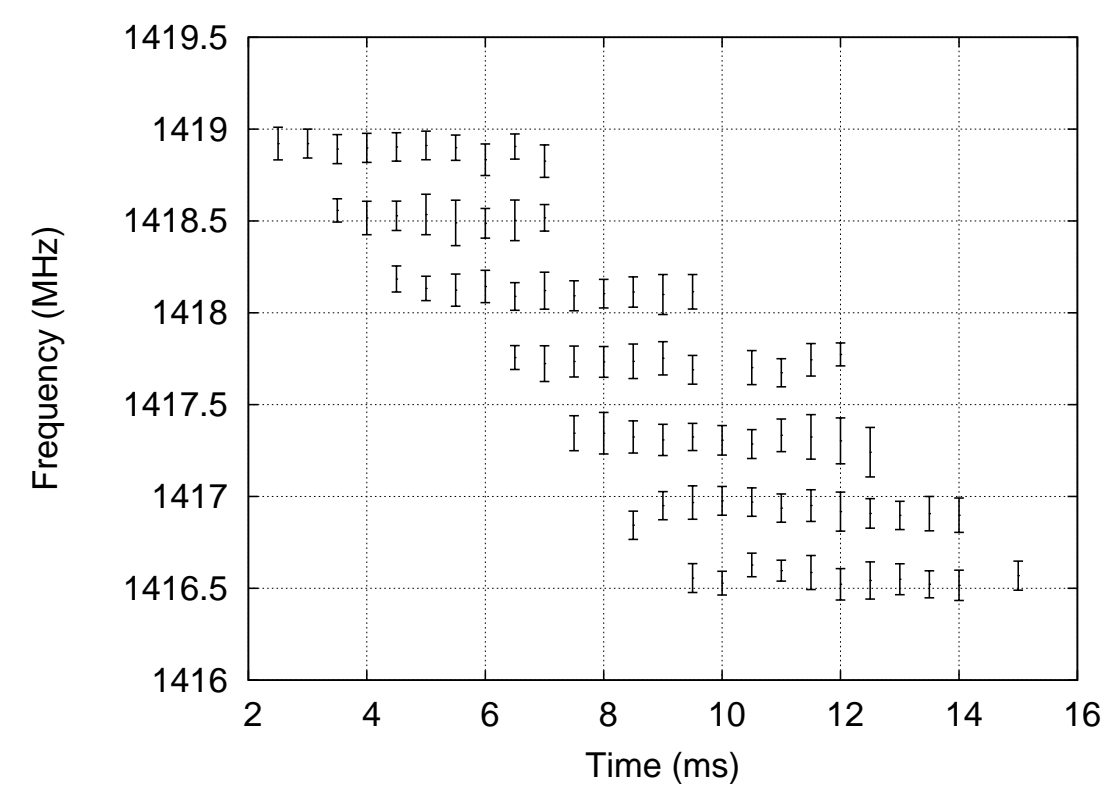

Figure 4. The locations of the centers of the Gaussian fits to the emission blobs for the episodes of emission observed at 19:44:13.13 are shown. The width of the best fit Gaussian is shown by the error-bars. The fitting error in the location of the Gaussian peak is usually smaller than the thickness of the line used to draw the symbols.

and a lower limit on the brightness temperature estimate of order $10^{12} \mathrm{~K}$ for this unresolved source.

We looked for evidence of a spectral slope in the individual emission blobs in an episode. A Gaussian was found to be a good representation for the spectral profile for the individual blobs of emission in a given episode. A Gaussian was fit to each of the emission blobs of an episode for every time slice and the location of the center of the Gaussian was used as the reference point to examine the slopes. The results of this analysis for one of the episodes of emission are shown in Figure 4. A vast majority of the blobs were fit very well with Gaussians. Occasionally the profile of adjacent Gaussians were broad enough to have a significant overlap and very infrequently, 2-3 times in an episode, the profile had significant asymmetry and was not fit. It is evident from this figure that, for a given emission blob, a straight line fit to the peaks of the best fit Gaussians would have a spectral slope close to $0 \mathrm{MHz}$ $\mathrm{s}^{-1}$. Our reference point, the peak of the best fit Gaussian wanders around by 2-3 spectral channels over the course of the emission blob. We believe that this wander is real and not the consequence of a poorly determined fit.

For the 19:44:13.13 episode of emission, where all the emission blobs are clearly defined we tried to obtain an average time profile. The analysis 

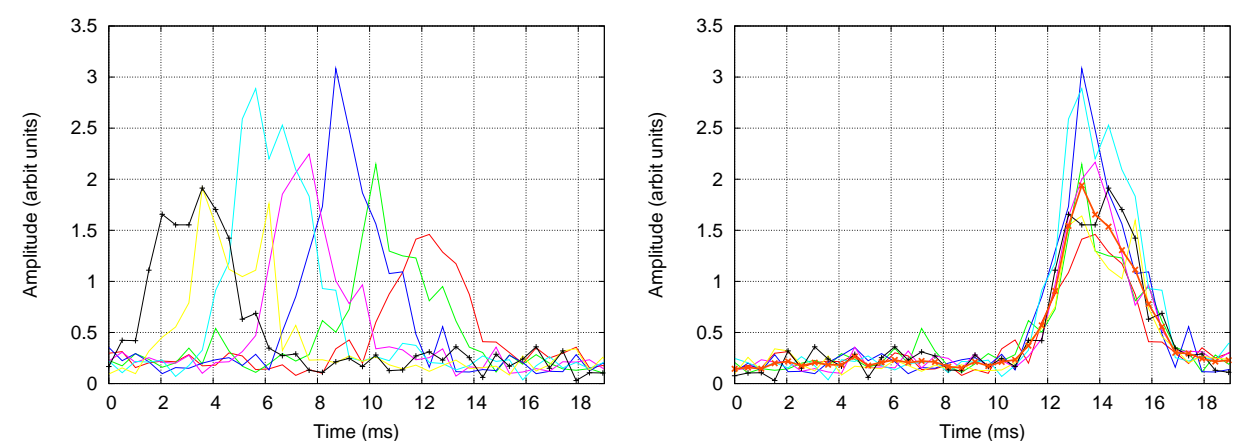

Figure 5. The left panel shows the time profiles averaged over three spectral channels, for each of the blobs of emission for the episodes of emission at 19:44:13.13. The right panel shows the same profiles after they have been aligned in time by compensating for the spectral slope across the emission band. The thick curve represents the average profile for the episode.

above was used to identify the spectral channel which is closest to the peaks of the Gaussian fits for the most time slices. The data from this and the two adjacent channels was coherently averaged to produce an average time profile for each emission blob and are shown in Figure 5. While the individual profiles differ in details, their widths and the time scales at which they rise and decay match very well. The rise is sharper than the decay, as would be expected in presence of scattering.

As has been mentioned earlier, an important advantage of interferometric observations is that they can be used to image radio emission and provide information about the location and morphology of the source. While imaging requires the use of an interferometer comprising multiple antennas, the interferometric phase from a single baseline observations can, in principle, be used to learn about the structure and evolution of the source of emission. The measured interferometric phase is the sum of astronomical and instrumental contributions. In order to extract the astronomical information from the phase, the instrumental contribution to the phase needs to be determined and subtracted. In absence of proper phase calibration, we estimate the instrumental component of phase variations across frequency and time using indirect means. The typical stability performance of the GPS receivers suggests a phase drift of $5^{\circ} \mathrm{s}^{-1}$. The spectral phase across the rather narrow band SRT receiver is expected to be fairly flat. This was verified using data from a different solar event observed on a different day which lasted $\approx 50 \mathrm{~ms}$ and showed emission covering the entire band. Under the assumption that the emission came from a stationary source close to the center of the delay beam, these data lead to an intrinsic instrumental phase ramp of $1.96 \pm$ $0.35^{\circ} \mathrm{MHz}^{-1}$. For the narrow band emission structure sweeping in time 

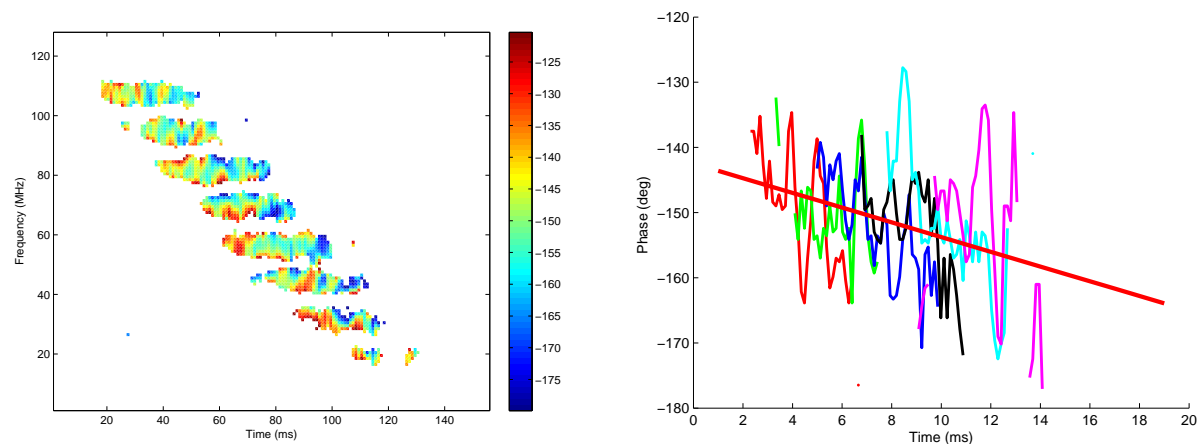

Figure 6. The left panel shows the phase for the episode seen at 19:44:13.13 in degrees. The phase of the parts of the band with low amplitude have been blanked out to make the phase variations across the emission blobs more evident. The right panel shows the phase measured for one of the spectral channels close to the center of the six of the emission blobs. The thick red line is the best fit straight line to the data plotted and has a slope of $-1.13 \pm 0.21^{\circ} \mathrm{ms}^{-1}$.

under discussion here, this phase slope will appear as a temporal slope of $0.6^{\circ} \mathrm{ms}^{-1}$. Another instrumental effect one needs to consider is that if the centroid of the emission source is not at the center of the delay beam of the interferometer, it leads to phase ramp across the band. For our observing setup a source at the limb of the Sun will lead to a phase ramp of $5.5^{\circ}$ $\mathrm{MHz}^{-1}$, which will appear like a drift in time of $\approx 1.65^{\circ} \mathrm{ms}^{-1}$. Another useful number is that a source moving with the speed of light perpendicular to the line of sight is going to produce a phase drift of $\pm 3.4^{\circ} \mathrm{ms}^{-1}$, for our setup.

For our case of an unresolved point source, the variation in the astronomical phase can be related to the change in the location of the source in the sky plane. Figure 6 shows the observed phase for the episode seen at 19:44:13.13 and highlights the variation in the phase across the episode. As shown in the right panel of Figure 6, though there is considerable variations in the phase observed across an emission blob, there does seem to be a general trend towards a small negative slope in this and most other episodes which were examined. This trend was quantified by fitting a straight line to the data. The large residuals to the straight line fit lead to the rather large errors on the fit parameters. The spread of residuals is consistent with the expectations for phase scatter for the $\approx 40$ SFU strength of the episodes of emissions and the signal to noise expectations. The slopes for emission episodes 2, 3, 6 and 11 (Table II) were determined to be $-1.13 \pm 0.21^{\circ}$ $\mathrm{ms}^{-1},-1.54 \pm 0.40^{\circ} \mathrm{ms}^{-1},-1.65 \pm 0.28^{\circ} \mathrm{ms}^{-1}$ and $0.30 \pm 0.38^{\circ} \mathrm{ms}^{-1}$, 
respectively. We do not regard these best fit slopes as being statistically significant. If interpreted as arising due to the motion of the centroid of the emission, a slope of $1.5^{\circ} \mathrm{ms}^{-1}$ implies a plane of the sky speed of $\approx 0.4 c$.

The phases averaged across entire episodes shows a variation of $5.7 \pm 1.1^{\circ}$ $\mathrm{s}^{-1}$ across the $\approx 8 \mathrm{~s}$ span of the episodes listed in Table II, close to the $\approx 5^{\circ}$ $\mathrm{s}^{-1}$ expected due to the clock drift. This suggests that the phase change due to the motion of the source in the plane of the sky is at most of the same order of magnitude as the clock drift, leading to an upper limit of $\approx 0.01 c$ on the plane of sky motion of the source.

\section{Discussion and Conclusions}

In our search of the literature, we did not come across any observations of bursts with similar temporal and spectral structure at nearby frequencies. The closest analogues, in terms of frequency-time structure, we discovered were reported by McConnell and Ellis in the early 1980s (McConnell et al., 1981; McConnell, 1982; McConnell, 1983) and were in the frequency range 30-80 MHz. They used the Llanherne radio telescope, a transit instrument which observed the sun for $10 \mathrm{~min}$ daily. On five occasions during three years of continuous operation and on additional three occasions during later intermittent operations, 1 to $2 \%$ of the recorded bursts were observed to have a similarly heavily frequency-modulated appearance. They reported seeing up to 16 regularly spaced emission blobs, which they referred to as fringes, spanning 5-13 MHz. The durations of fringes ranged between 20 and $140 \mathrm{~ms}$, with a mean of about $50 \mathrm{~ms}$ and the drift rates ranged between -1.5 and $-5 \mathrm{MHz} \mathrm{s}^{-1}$. The fractional bandwidth of the fringes was of order $10^{-3}$. The fringe separation increased with frequency from $\approx 60 \mathrm{kHz}$ at 30 $\mathrm{MHz}$ to $\approx 90 \mathrm{kHz}$ at $40 \mathrm{MHz}$ and they were not seen above $57 \mathrm{MHz}$. Barrows et al. (1984) reported observations of bursts with similar morphologies and characteristics at meter wavelengths.

Millisecond radio spikes have been reported in the literature for decades and they have been studied extensively (Benz, 1986; Benz and Kane, 1986; Güdel and Benz, 1990; Messmer et al., 2000) and Benz (2004) provides an excellent recent review of decimetric burst emission. Dạbrowski et al. (2005) presented a detailed report on narrow-band ms radio spikes in the decimeter band observed using a $15 \mathrm{~m}$ telescope in Poland with a spectral and temporal resolution of $3 \mathrm{MHz}$ and $80 \mu \mathrm{s}$, respectively. These bursts are defined somewhat arbitrarily to have individual emissions no longer than $100 \mathrm{~ms}$ and observed above $1 \mathrm{GHz}$. They usually appear in groups of many thousands, have flux densities of order $100 \mathrm{SFU}$ and brightness temperatures greater than $10^{13} \mathrm{~K}$ to $10^{15} \mathrm{~K}$. Their fractional bandwidths tend to be of order a few percent and vary significantly from one event to another within the 
same group. They also tend to be rather infrequent and only 13 events have been found in 1990.4 hours of observations spread over 357 observing days. More recently Dąbrowski and Kus (2007) show some events whose frequencytime-plane structure is similar to a scaled-up version of the bursts presented here; their spectral widths of the individual emission blobs are $\approx 5 \mathrm{MHz}$, and the temporal widths are $\approx 30 \mathrm{~ms}$. Though they have sufficient time resolution to capture such events, the dilution of the signal due to their coarse bandwidth and inability to reject the extended emission from the Sun reduces their sensitivity to these events.

The $10^{12} \mathrm{~K}$ brightness temperature implies that this emission must come from a coherent emission mechanism. The two well-accepted coherent emission mechanisms, in context of solar emission, are plasma emission and electron-cyclotron maser emission. The former is believed to be the mechanism responsible for Type II and III solar burst emission and leads to emission at the local plasma frequency and its harmonic (e.g. Dulk, 2000; Gopalswamy, 2000; Robinson and Cairns, 2000), and the latter is used to explain the milli-second solar spikes and and bright radio sources observed during impulsive phase of solar flares (e.g. Melrose and Dulk, 1982; Willes and Robinson, 1996).

Assuming this emission to be plasma emission allows one to use the observed drift rate and a model for coronal electron density to arrive at coronal height and velocity for these electrons, $v_{\mathrm{e}}$. Using the Newkirk model for the quiet sun (Newkirk, 1961) McConnell (1983) arrived at velocities in the range $0.05 c<v_{\mathrm{e}}<0.1 c$. Using the Newkirk model for electron density distribution over active regions for our observations (instead of that for the quiet sun used by McConnell (1983)), a local plasma frequency of 1420 $\mathrm{MHz}$ implies a local electron density, $n_{\mathrm{e}}$ of $2.5 \times 10^{10} \mathrm{~cm}^{-3}$, which exceeds the density over active regions at the base of the photosphere extrapolated from the Newkirk model by about a factor of $\approx 15$ (assuming the emission to be at the harmonic rather than the fundamental reduces it to a factor of $\approx 3.5$ ). In general, the high densities implied by these and other even higher frequency decimetric type-III-like bursts are yet to find a convincing explanation (Benz, 2004). A very large X-ray flare had been observed 22 min prior to these observations and a large CME was seen by the LASCO coronagraph 16 min after this event. At such times, it is not unreasonable to expect the solar corona to have considerable departures from stable models and be in a rapid state of evolution. If the emission is at the fundamental, the presence of a limited scattering signature implies that this radiation did not travel through much scattering medium, suggesting that its source might be an over-dense region high up in the corona. Small-scale magnetic reconnection, which seems to be ubiquitous on the sun, is the most probable source for these energetic electrons. 
This event has much finer temporal and spectral structure, and is much more monochromatic, as compared to the so called Zebra bursts which have been reported in the literature by various groups (e.g Elgaroy, 1959; Slottje 1972; Chernov et al., 2003). In spite of a large number of studies, many important questions about their origin and mechanism still remain unsettled. Various options have been proposed including a cyclotron maser model (Winglee and Dulk, 1986; LaBelle et al., 2003), an interference mechanism (Barta and Karlicky, 2006; Ledenev et al., 2006) and non-linear coupling of Bernstein waves (Altyntsev et al., 2005).

In conclusion, we report very high temporal and spectral resolution interferometric observations of some unusual decimetric radio bursts. They were observed 22 minutes after a GOES class X flare and comprise mutiple episodes of emission, each of which is heavily frequency-modulated with a spectral slope of about $-245.5 \mathrm{MHz} \mathrm{s}^{-1}$ and lasts for $10-15 \mathrm{~ms}$. The constituent blobs of emission for each of these episodes have brightness tempeatures in the range of $10^{12} \mathrm{~K}$, are only $200-250 \mathrm{kHz}$ wide, appear every $\approx 400 \mathrm{kHz}$, do not seem to have any spectral slope and last for $4-$ $5 \mathrm{~ms}$. The light travel time argument and detection by an interferometer with sub-arcmin resolution require that this emission must come from a very compact region on the Sun. The very high brightness temperature of the emission and its highly monochromatic nature both imply that coherent emission mechanisms must be at play. The very high temporal and spectral resolution needed to detect these bursts highlights that this is a poorly explored part of the phase space. While the data available for this study lack the signal-to-noise ratio to make strong statements about the motion of the centroid of emission, it does highlight the promise of interferometric observations to provide unique and detailed information about such events. We hope that these observations will interest some experts in the field to attempt to explain and interpret them in detail.

\section{Acknowledgements}

The authors thank John F. C. Wardle and David Roberts for their suggestion to examine the dynamic spectra in detail, which lead to the discovery of this event in the data. We acknowledge Dale Gary for providing the data from Owens Valley Solar Array, a part of the Owens Valley Radio Observatory and the anonymous referees for their constructive suggestions, responding to which has resulted in significant improvements to the work presented here. This work was supported by the National Science Foundation Research Experience for Undergraduates (REU) program under grant AST-0138506 to the MIT Haystack Observatory. The authors thank Haystack Observatory staff for their assistance in setting up and operating the SRT interferometer. 


\section{References}

Altyntsev, A. T., Kuznetsov, A. A., Meshalkina, N. S., Rudenko, G. V., Yihua Yan: 2005, Astron. Astrophys. 431, 1037.

Barrows, C. H., Flagg, R. S., Perrenoud, M.: 1984, Solar Phys. 90, 111.

Barta, M., Karlicky, M.: 2006, Astron. Astrophys. 450, 359.

Benz, A. O.: 1986, Solar Phys. 104, 99.

Benz, A. O., Kane, S. R.: 1986, Astron. Astrophys. 104, 179.

Benz, A. O., Graham, D., Isliker, H., Andersson, C., Koehnlein, W., Mantovani, F., Umana, G.: 1996, Astron. Astrophys. 305, 970.

Benz, A. O.: 2004, In: Gary, D.E., Keller, C.U. (eds.), Solar and Space Weather Radiophysics: Current Status and Future Developments, Kluwer Academic Publishers, Dordrechet, 203.

Chernov, G. P., Yan, Y. H., Fu, Q. J.: 2003, Astron. Astrophys., 406, 1071.

Dąbrowski, B. P., Rudawy, P., Falwwicz, R., Siarkowski, M., Kus, A. J.: 2005, Astron. Astrophys. 434, 1139.

Dạbrowski, B. P, Kus, A. J.: 2007: Mem. Soc. Astron. Ital.78, 264.

Dulk, G. A.: 2000, In : Stone, R.G., Weiler K.W., Godlstein, W.L., Bourgeret, J.-L. (eds.),Radio Astronomy at Long Wavelengths, AGU Geophys. Monogr. 119, 115.

Elgaroy, O.: 1959, Nature 184, 887.

Gary, D.E.: 2003, In: Oswalt, T.D. (ed.), The Future of Small Telescopes in the New Millennium II. The Telescopes We Use, Kluwer Academic Publishers, Dordrecht, 309.

Gopalswamy, N.: 2000, In : Stone, R.G., Weiler K.W., Godlstein, W.L., Bourgeret, J.-L. (eds.), Radio Astronomy at Long Wavelengths, AGU Geophys. Monogr. 119, 123.

Güdel, M., Benz A. O.: 1990, Astron. Astrophys. 231, 202.

LaBelle, J., Truemanm, R. A., Yoon, P. H.: 2003, Astrophys. J. 593, 1195.

Ledenev, V. G., Yan, Y., Fu, Q.: 2006, Solar Phys. 233, 129L.

McConnell, D., Ellis, G. R. A.: 1981, Solar Phys. 69, 161.

McConnell, D.: 1982, Solar Phys. 78, 253.

McConnell, D.: 1983, Solar Phys. 84, 361.

Melrose, D. B., Dulk, G. A.: 1982, Astrophys. J. 259, 844.

Messmer, P., Benz, A. O.: 2000, Astron. Astrophys. 384, 287.

Newkirk, G., Jr.: 1961, Astrophys. J. 133, 983.

Robinson, P. A., Cairns, I. H.: 2000, In : Stone, R.G., Weiler K.W., Godlstein, W.L., Bourgeret, J.-L. (eds.),Radio Astronomy at Long Wavelengths, AGU Geophys. Monogr. 119, 27.

Salah, J,E., Pratap, P., Rogers, A. E. E.: 2003, In: Oswalt, T.D. (ed.), The Future of Small Telescopes in the New Millennium II. The Telescopes We Use, Kluwer Academic Publishers, Dordrecht, 323.

Slottje, C.: 1972, Solar Phys. 25, 210.

Tapping, K. F.: 1986, Solar Phys. 104, 199.

Tapping, K. F., Kuijpers, J., Kaastra, K. S., van Niewkoop, J. , Graham, D., Slottje, C. L.: 1986, Astron. Astrophys. 122, 177.

Thompson, A. R., Moran, J. M., Swenson, G. W., Jr.: 1986, Interferometry and Synthesis in Radio Astronomy, John Wiley \& Sons, New York, 217.

Willes, A. J., Robinson, P. A.: 1996, Astrophys. J. 467, 465.

Winglee, R. M., Dulk, G. A.: 1986, Astrophys. J. 307, 808. 
solar-phy.tex; 22/09/2009; 10:34; p.16 